\title{
Characterization of the Mechanisms by which Gelatinase A, Neutrophil Collagenase, and Membrane-Type Metalloproteinase MMP-14 Recognize Collagen I and Enzymatically Process the Two $\alpha$-Chains
}

\author{
Magda Gioia ${ }^{1,2}$, Susanna Monaco ${ }^{1,2}$, Giovanni Francesco Fasciglione ${ }^{1}$ \\ Anna Coletti ${ }^{1}$, Andrea Modesti ${ }^{1}$, Stefano Marini ${ }^{1}$ \\ and Massimo Coletta ${ }^{1,2 \star}$
}

\author{
${ }^{1}$ Department of Experimental \\ Medicine and Biochemical \\ Sciences, University of Roma \\ Tor Vergata, Via Montpellier 1 \\ I-00133 Rome, Italy \\ ${ }^{2}$ InterUniversity Consortium for \\ the Research on the Chemistry of \\ Metals in Biological Systems \\ (CIRCMSB), P.za Umberto I 1 \\ I-87100 Bari, Italy
}

\begin{abstract}
The turnover of native collagen has been ascribed to different members of the matrix metalloproteinase (MMP) family. Here, the mechanisms by which neutrophil collagenase (MMP-8), gelatinase A (MMP-2), and the ectodomain of MT1-MMP (ectMMP-14) degrade fibrillar collagen were examined. In particular, the hydrolysis of type I collagen at $37{ }^{\circ} \mathrm{C}$ was investigated to identify functional differences in the processing of the two $\alpha$-chain types of fibrillar collagen. Thermodynamic and kinetic parameters were used for a quantitative comparison of the binding, unwinding, and hydrolysis of triple helical collagen. We demonstrate that the MMP family has developed at least two distinct mechanisms for collagen unwinding and cleavage. MMP-8 and ectMMP-14 display a similar mechanism (although with different catalytic parameters), which is characterized by binding (likely through the hemopexin-like domain) and cleavage of $\alpha-1$ and/or $\alpha-2$ chains without distinguishing between them and keeping the gross conformation of the triple helix (at least during the first cleavage step). On the other hand, MMP-2 binds preferentially the $\alpha-1$ chains (likely through the fibronectin-like domain, which is not present in MMP-8 and ectMMP-14), grossly altering the whole triple helical arrangement of the collagen molecule and cleaving preferentially the $\alpha-2$ chain. These distinctive mechanisms underly a drastically different mode of interaction with triple helical fibrillar collagen I, according to which the MMP domain is involved in binding. These findings can be related to the different role exerted by these MMPs on collagen homeostasis in the extracellular matrix.
\end{abstract}

(C) 2007 Elsevier Ltd. All rights reserved.

Keywords: collagen I; cleavage; binding; circular dichroism; kinetics

\section{Introduction}

The collagen family represents a group of different extracellular matrix proteins, which provide strength and rigidity to the connective tissue

Abbreviations used: MMP, matrix metalloproteinase; MT-MMP, membrane-type matrix metalloproteinase; $\mathrm{CBD}$, collagen binding domain; ectMMP-14, ectodomain of MMP-14.

E-mail address of the corresponding author: coletta@seneca.uniroma2.it due to the presence of a particular quaternary structure (called the triple helix structure). ${ }^{1}$ Fibrillar collagens have the capacity to self-associate, generating a supramolecular organization of $67 \mathrm{~nm}$ D periodic fibrils. 2,3 In particular, its monomer consists of three left-hand $\alpha$-chains that are coiled around each other into a right-hand triple helix. This characteristic folding requires a repeated sequence, that is Gly-X-Y, where $X$ and $Y$ positions are mainly proline and 4-trans-L-hydroxyproline residues, respectively. ${ }^{4}$ This assembly is particularly observed in fibrillar type I collagen, which is one of the main components of the extracellular matrix. ${ }^{1,3}$ 
Collagen matrix degradation and remodeling are important aspects of normal tissue homeostasis, because the alteration of collagen turnover is often associated with the progression of diseases like fibrosis, arthritis, cancer, atherosclerosis, and aneurysm. ${ }^{5-8}$ Moreover, the degradation of extracellular collagen drives cell migration, proliferation, and differentiation through the uncovering of collagen cryptic molecular determinants. ${ }^{9-11}$

The complex triple helical fold renders collagen extremely resistant to proteolytic attack and only a few classes of proteases are able to degrade this molecule; among them, the most prominent members belong to the matrix metalloproteinases (MMPs) family.

The matrix metalloproteinases are a family of $\mathrm{Ca}^{2+}$ and $\mathrm{Zn}^{2+}$-dependent neutral endopeptidases collectively capable of degrading essentially all components of the extracellular matrix. The MMPs, which are synthesized by connective tissue cells, are important for the remodeling of the extracellular matrix that accompanies physiologic processes (such as uterine involution, bone resorption, and wound healing) as well as pathologic processes (such as tumor growth and diffusion). ${ }^{12}$ This wide spectrum of substrates and activities is reflected in the complexity of their recognition and degradation mechanisms.

MMPs are multidomain enzymes and they can be further subdivided (according to their domain composition and their efficiency in degrading substrates) into five groups, namely: (a) matrilysins, (b) collagenases (e.g., MMP-8), (c) gelatinases (e.g., MMP-2), (d) stromelysins, and (e) membrane-type MMPs (e.g., MMP-14). ${ }^{13}$

The general structure of MMPs includes a signal peptide, a propeptide domain, a catalytic domain with a highly conserved zinc-binding active site, and a hemopexin-like domain. In particular, collagenases, as well as the soluble form of members of membrane-type (MT)-MMP group, display a catalytic domain linked to the hemopexin-like domain via a short hinge peptide, whereas gelatinases (namely MMP-2 and MMP-9) also contain fibronectin type II inserts (consisting of three tandem copies of the fibronectin type II-like module) within a loop in the catalytic domain. Further, whole MT-MMPs contain at the C-terminal end of the hemopexin-like domain a transmembrane domain, which can be cleaved off under physiological conditions, bringing about the shedding of soluble forms in the extracellular matrix. ${ }^{13,14}$ In the past few years it has been reported that, beside the catalytic site, regions far from the active site (called exosites) play an important role in facilitating both substrate binding and proteolysis. $^{15}$

Within the MMP family only three subclasses (namely collagenases, gelatinases, and MT-MMPs) are involved in native fibrillar collagen degradation. ${ }^{16-19}$ In view of their importance, these enzymes have been deeply studied in the past 20 years. In particular, most of the information on their enzymatic mechanism was elucidated by studies on triple helix peptides, illustrating that gelatinases possess distinct features with respect to collagenases and MT-MMPs. ${ }^{20-24}$

On the other hand, only few data are available on the MMP mechanisms of cleaving native collagens; among them, we must mention the comparison of collagenolytic activity at $25^{\circ} \mathrm{C}$ between MMP-2 and MMP- $14,{ }^{23}$ the study of the unwinding properties of MMP-1, ${ }^{25}$ and the enzymatic mechanism of MMP-8 on fibrillar collagen at $37{ }^{\circ} \mathrm{C} .{ }^{26,27}$ Nevertheless, accurate mechanisms for binding, distorting, and hydrolyzing triple helical structures by these three class of MMPs are still far from being understood, mainly because of the lack of structural information on fibrillar collagen. However, very recently some structural information on fibrillar collagen has become available, ${ }^{3}$ confirming that the substratebinding site of MMPs is too narrow to accommodate triple helical collagen, as already postulated on the basis of the three-dimensional structural data of several MMPs. ${ }^{15,25,28-30}$ As a consequence, it has been recently supposed that MMP exosites may induce at least a local unwinding of the triple helix to allow every collagenolytic step to take place. ${ }^{21,31,32}$

Several factors contribute to rendering the elucidation of the mechanism of collagen processing by MMPs a very challenging task. First of all, we must mention that collagen structure is temperature dependent (with a melting point close to the physiological temperature of $37^{\circ} \mathrm{C}$ ) and the low thermostability of collagen was suggested to be important for the independent mobility of individual helices required for various biological functions. ${ }^{33}$ NMR studies have shown that at $37^{\circ} \mathrm{C}$ triple helices have backbone motilities even in fibrils, ${ }^{34}$ and it has been established that the energetically preferred conformation of type I collagen is more toward a random coil rather than toward a compact triple helix. ${ }^{35} \mathrm{~A}$ second point is represented by the fact that little information is available on the rearrangements that occur upon the MMP-collagen interaction to catalyze each hydrolysis of the three cleavable peptide bonds, leading to the formation of the $1 / 4$ and $3 / 4$ fragments. ${ }^{23,25,36}$

To contribute to some of the points above, we compared at $37^{\circ} \mathrm{C}$ the hydrolysis of native collagen I by neutrophil collagenase MMP-8, gelatinase A MMP-2, and ectMMP-14, aiming to describe quantitatively the different mechanisms by which these MMPs interact with and enzymatically process the two types of $\alpha$-chains of fibrillar type I collagen.

The present study allows the assertion that the MMP family has evolved at least two different mechanism in processing collagen type I: MMP-2 proteolytic activity requires a substantial unwinding of the collagen I molecule, whereas the cleavage process by MMP- 8 and ectMMP-14 can occur on an almost intact triple helix. In addition, the preferential enzymatic action on the $\alpha-2$ chain by MMP-2, which is not observed in the case of MMP-8 and ectMMP-14, suggests that as long as the triple helical structural arrangement is maintained, no preferential enzymatic action is exerted on either of the two 
types of chains. This information should represent an important step toward the elucidation of the molecular determinants controlling collagen catabolism and they could be used to address new therapeutic strategies to modulate collagen destruction in degenerative diseases.

\section{Results}

We have previously shown a marked temperature dependence for the intrinsic enzymatic activity of MMP-2, MMP-9, and MMP-8 37 and for the proteolytic activity of MMP-8 on natural substrate. ${ }^{27}$ Because most of the biochemical studies on the enzymatic activity of MMPs have been performed at $25{ }^{\circ} \mathrm{C}^{21,23-25,32}$ and the biological relevance of collagen proteolysis at $37{ }^{\circ} \mathrm{C}$ has been reported in several cell culture experiments, ${ }^{18,38}$ we decided to carry out an investigation on collagen I degradation by MMP-2, MMP-8, and ectMMP-14 at $37^{\circ} \mathrm{C}$, under conditions as close as possible to the physiological conditions. Obviously, at $37^{\circ} \mathrm{C}$ the structure of the collagen triple helix is different from that observed at $25^{\circ} \mathrm{C}$, as previously reported on the basis of $\mathrm{CD}$ ellipticity degree at $223 \mathrm{~nm} \cdot{ }^{27}$ As a consequence, after $3 \mathrm{~h}$ of incubation of collagen with trypsin at $37{ }^{\circ} \mathrm{C}$ a moderate enzymatic processing can be observed, which starts only after $2 \mathrm{~h}$ (Figure 1, lane 3). However, this result does not imply that at $37^{\circ} \mathrm{C}$ the structure is less "native" than at lower temperatures, as long as whatever happens to the triple helical assembly is fully reversible. Therefore, to validate this occurrence, we incubated collagen I at $37^{\circ} \mathrm{C}$ for $24 \mathrm{~h}$ and then brought the sample back to $28{ }^{\circ} \mathrm{C}$ (the highest temperature reported in the literature). Subsequent exposure to trypsin did not show any evidence of enzymatic processing (Figure 1 , lane 2), thus demonstrating that at $37^{\circ} \mathrm{C}$ the

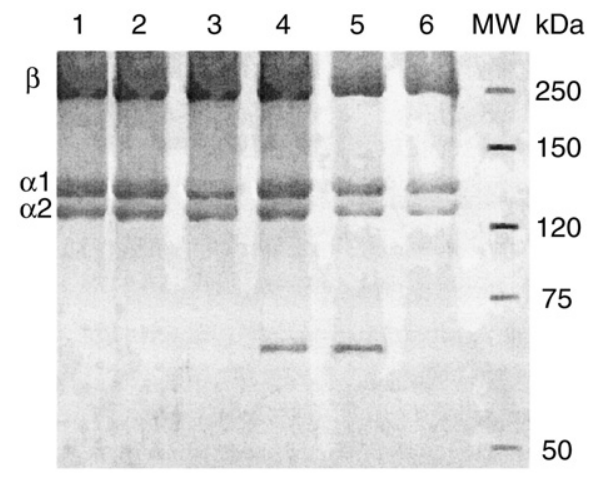

Figure 1. SDS-(7.5\%) PAGE electrophoresis of collagen I degradations. In the absence of any enzyme (lane 1), by trypsin $(10 \mathrm{nM})$ for $3 \mathrm{~h}$ at $28{ }^{\circ} \mathrm{C}$ after $24 \mathrm{~h}$ incubation at $37^{\circ} \mathrm{C}$ (lane 2$)$, by trypsin $(10 \mathrm{nM})$ for $3 \mathrm{~h}$ at $37^{\circ} \mathrm{C}$ (lane 3$)$, by MMP-2 $(2 \mu \mathrm{M})$ fully inhibited by Batimastat for $3 \mathrm{~h}$ (lane 4$)$, by trypsin $(10 \mathrm{nM})$ for $3 \mathrm{~h}$ in the presence of MMP$2(2 \mu \mathrm{M})$ fully inhibited by Batimastat (lane 5), and by active MMP-2 (10 pM) for $3 \mathrm{~h}$ at $37^{\circ} \mathrm{C}$ (lane 6). Lane 7 reports MW markers. For further details, see the text. conformation is somewhat looser, as expected, but still native, as indicated by the full reversibility with temperature of the compactness of the triple helix. Importantly, under these conditions, in the presence of collagen all three MMPs are perfectly stable without undergoing any autocatalytic activity over $60 \mathrm{~min}$ at $37^{\circ} \mathrm{C}$ (data not shown), which is the period needed to obtain kinetic data for the calculation of catalytic parameters.

\section{The catalytic action of MMP-2, MMP-8, and ectMMP-14 on $\alpha-1$ and $\alpha-2$ chains}

The degradation of collagen occurs through an ordinate series of cleavage events ${ }^{26}$ and the first step (corresponding to the formation of $3 / 4$ and $1 / 4$ fragments) ${ }^{36}$ is crucial because of the fairly slow rate of collagen turnover within the cartilage. ${ }^{39}$ We obtained catalytic parameters on each $\alpha$-chain (i.e., $k_{\text {cat }} / K_{\mathrm{m}}, k_{\text {cat, }}$ and $\left.K_{\mathrm{m}}\right)$ for the three MMPs by following the time dependence of substrate disappearance as a function of substrate concentration under nondenaturing conditions to sort out the action of MMPs on the two different chains of collagen I.

Figure 2 shows an example of the electrophoretic pattern of collagen I degradation observed for each MMP investigated. In the gels we can see three bands (identified as $\beta, \alpha-1$, and $\alpha-2$ ), which change intensity as a function of time of incubation with MMP-2, MMP-8, and ectMMP- 14 at $37^{\circ} \mathrm{C}$. Although these three species have never been deeply characterized, there is a general agreement that $\alpha-1$ and $\alpha-2$ forms are the two types of chains, forming the actual triple helical collagen I molecule (in a 2:1 ratio), while the $\beta$ form is a cross-linked polymer of fibrils formed by two triple helical collagen I molecules linked through intermolecular telopeptide interactions and interlysyl bonds. ${ }^{1,40}$

We followed the time evolution for the first hour of the band intensity for all three species after incubation with each MMP at $37^{\circ} \mathrm{C}$, determining the enzymatic processing only for the two chains (and not for the $\beta$ form) at different concentrations of collagen I. The apparent cleavage rate was measured at each employed concentration of collagen and it was normalized to the amount of active enzyme. As expected, in all cases the resulting reciprocal of the steady-state velocity as a function of the reciprocal of $\alpha-1$ and $\alpha-2$ concentration is linear (Figure 3) and this evidence indicates that, at least for the first step, the enzymatic cleavage of $\alpha-1$ and $\alpha-2$ chains of collagen I follows the MichaelisMenten mechanism. The derived parameters $k_{\text {cat }}$ $K_{\mathrm{m}}$, and $k_{\text {cat }} / K_{\mathrm{m}}$ are reported in Table 1.

Looking at the parameters for both $\alpha$ chains reported in Table 1, it appears clear that MMP-2, which is characterized by weak collagenase activity at $25^{\circ} \mathrm{C}, 15$ shows powerful collagenase activity at the physiological temperature of $37^{\circ} \mathrm{C}$, even higher than the collagenase itself (i.e., MMP-8). However, this observation is not unexpected in view of the reported very high temperature dependence (corresponding 


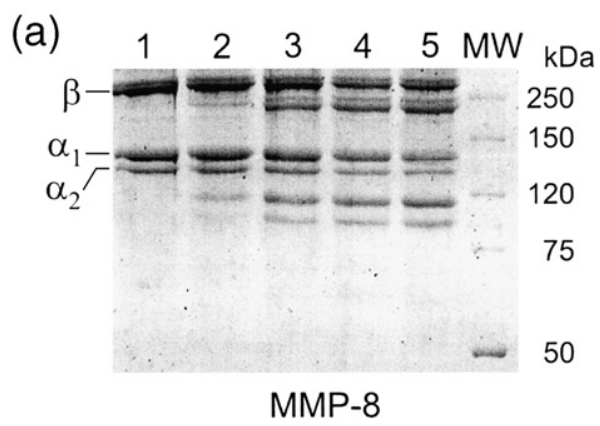

(b)
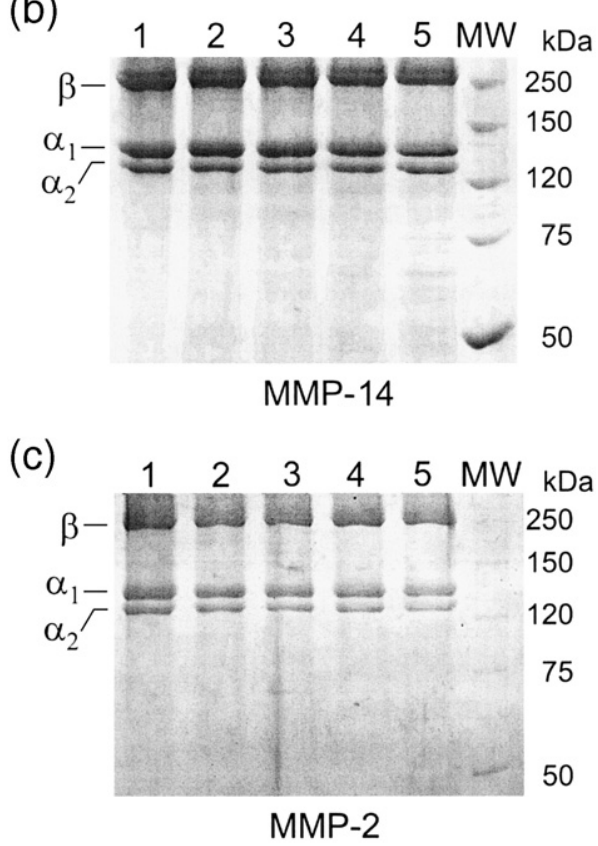

Figure 2. SDS-(7.5\%) PAGE (7.5\%) electrophoresis of the degradation kinetics of bovine collagen I at $37^{\circ} \mathrm{C}, \mathrm{pH}$ 7.3, by MMP-8 (a), ectMMP-14 (b), and MMP-2 (c). Different collagen chains are indicated; the incubation time is: (for a and b) $0 \mathrm{~min}$ (lane 1), $20 \mathrm{~min}$ (lane 2), $40 \mathrm{~min}$ (lane 3), 50 min (lane 4), 60 min (lane 5); (for c): 0 min (lane 1), $10 \mathrm{~min}$ (lane 2), $20 \mathrm{~min}$ (lane 3), $30 \mathrm{~min}$ (lane 4), $40 \mathrm{~min}$ (lane 5). The last column reports MW markers. For further details, see the text.

to a high Arrhenius activation energy) for the catalytic behavior of MMP-2 compared to other MMPs. ${ }^{37}$

At $37{ }^{\circ} \mathrm{C}$ MMP-8 and ectMMP-14 show similar behavior for the two different chains both for $k_{\text {cat }}$ and for $K_{\mathrm{m}}$, suggesting that they do not distinguish between the two types of chains. However, some difference between MMP-8 and ectMMP-14 can be observed for the catalytic parameters, because MMP-8 shows a somewhat higher affinity for substrates, whereas ectMMP-14 appears somewhat faster for the cleavage rate-limiting step (see Table 1).

A drastic difference can be noticed for the action of MMP-2 on the different chains of collagen I at $37^{\circ} \mathrm{C}$. Thus, beside a much higher overall catalytic efficiency of MMP-2 with respect to MMP-8 and ectMMP-14, the value of $k_{\text {cat }} / K_{\mathrm{m}}$ is somewhat different for the two chains, being a little higher toward the $\alpha-2$ chain than the $\alpha-1$ chain (see Table 1 ). However, it must be stressed that this feature is connected to a different contribution of $k_{\text {cat }}$ and $K_{\mathrm{m}}$ for the different substrates. Thus, the slower $k_{\text {cat }} / K_{\mathrm{m}}$ for the $\alpha-1$ chain of collagen I is fully attributable to the very slow $k_{\text {cat }}$ (partially compensated by a very low value for $K_{\mathrm{m}}$, referrable to a strong interaction of MMP- 2 with the recognition site(s) on the $\alpha-1$ chain), while in the case of the $\alpha-2$ chain a very fast $k_{\text {cat }}$ is observed (see Table 1) with a fairly high $K_{\mathrm{m}}$ value. This feature clearly indicates a completely different modulation mechanism for substrate cleavage with respect to the partner chain $\alpha-1$, envisaging a different role of the two chains in the interaction with MMP-2. As a whole, this result indicates that
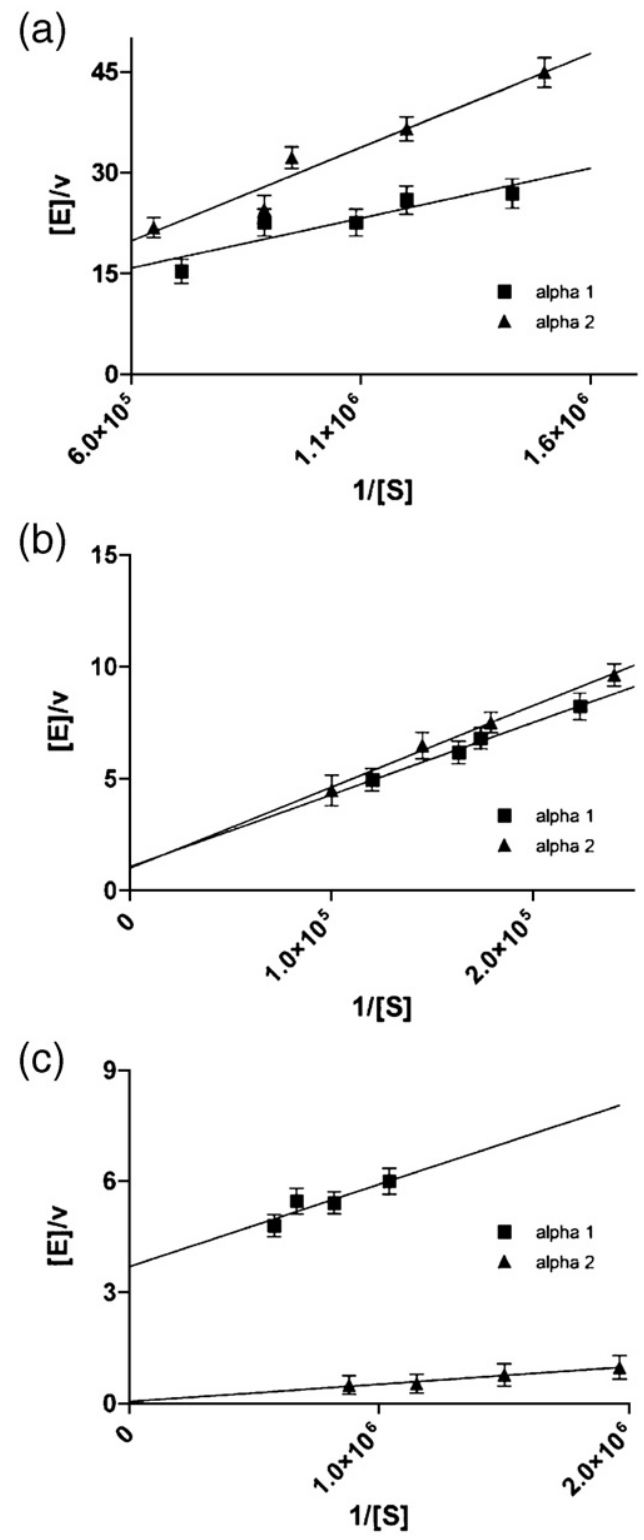

Figure 3. Lineweaver-Burk double-reciprocal plots of the steady-state velocity versus substrate concentration for the first-step cleavage of bovine collagen I by MMP-8 (a), ectMMP-14 (b), and MMP-2 (c). Solid lines are leastsquares fitting of data according to equation (1). For further details, see the text. 
Table 1. Catalytic parameters for the enzymatic cleavage of $\alpha-1$ and $\alpha-2$ chains of collagen I at $37^{\circ} \mathrm{C}$ by different MMPs

\begin{tabular}{lcccc}
\hline Enzyme & $\begin{array}{c}\text { Collagen } \\
\text { chain }\end{array}$ & $\begin{array}{c}k_{\text {cat }} / K_{\mathrm{m}} \\
\left(\mathrm{M}^{-1} \mathrm{~s}^{-1}\right)\end{array}$ & $k_{\text {cat }}\left(\mathrm{s}^{-1}\right)$ & $K_{\mathrm{m}}(\mathrm{M})$ \\
\hline MMP-8 & $\alpha-1$ & $5.0( \pm 0.6) \times 10^{4}$ & $0.32 \pm 0.05$ & $6.4( \pm 0.7) \times 10^{-6}$ \\
& $\alpha-2$ & $3.3( \pm 0.4) \times 10^{4}$ & $0.31 \pm 0.05$ & $9.4( \pm 1.1) \times 10^{-6}$ \\
ectMMP-14 & $\alpha-1$ & $3.1( \pm 0.4) \times 10^{4}$ & $1.05 \pm 0.12$ & $3.4( \pm 0.4) \times 10^{-5}$ \\
& $\alpha-2$ & $2.8( \pm 0.3) \times 10^{4}$ & $0.99 \pm 0.11$ & $3.5( \pm 0.4) \times 10^{-5}$ \\
MMP-2 & $\alpha-1$ & $4.5( \pm 0.5) \times 10^{5}$ & $0.27 \pm 0.03$ & $6.0( \pm 0.8) \times 10^{-7}$ \\
& $\alpha-2$ & $2.0( \pm 0.3) \times 10^{6}$ & $70.1 \pm 8.1$ & $3.5( \pm 0.4) \times 10^{-5}$ \\
\hline
\end{tabular}

MMP-2 preferentially interacts with the $\alpha-1$ chain, whereas the $\alpha-2$ chain is more susceptible to cleaveage.

\section{Unwinding activity}

Kinetics of collagen degradation was performed on the native triple helix and the analysis by SDSPAGE electrophoresis allows information to be obtained on each chain separately. Therefore, to combine the enzymatic action on the individual chains to the overall effect on the triple helix during the cleavage process we recorded the CD spectra of type I collagen during proteolysis by one of the three MMPs investigated.

\section{MMP-8 and ectMMP-14}

In the case of MMP- 8 very minor changes can be detected for the CD spectra over the time period during which the first cleavage step into the $1 / 4$ and $3 / 4$ fragments takes place, ${ }^{26}$ suggesting that the unwinding of the molecule, if any, is very limited. A similar behavior is also shown by ectMMP-14 (Figure 4(a)): in fact, at the beginning of the collagen I degradation process, no meaningful change was observed. Thus, also in this case, the unwinding of collagen by ectMMP-14 action during the first step eventually involves only a limited portion of the triple helix without perturbing the overall structure. Further, agarose-entrapped collagen I incubated with fully inhibited ectMMP-14 and MMP-8 did not show any variation of the CD spectrum (data not shown), clearly indicating that binding itself does not perturb significantly the triple helical structure.

An analogous behavior was previously suggested for collagenase MMP-1, proposing that the enzyme locally unwinds triple helical collagen before hydrolyzing the peptide bonds. ${ }^{25}$ On the other hand, after the first cleavage step further degradation of collagen I occurs by MMP-8 and ectMMP-14, accompanied by a large variation of the CD spectrum with a great decrease in peak intensity at 223-225 nm (see Figure $4(\mathrm{a})){ }^{26}$

\section{Gelatinase MMP-2}

Unlike MMP-8 and ectMMP-14, the exposure of the agarose gel-entrapped collagen I to MMP-2 brings about large CD spectral changes from the beginning with a rapid decrease of ellipticity (see Figure 4(b)). These results agree with that reported by others ${ }^{23}$ after the addition of the collagen binding domain (CBD) of MMP-2 to collagen I (which indeed interacts with collagen I without cleaving it), ${ }^{23}$ and they are grossly different from that shown by the other two MMPs, where the initial cleavage step is not accompanied by any relevant rearrangement on collagen structure (see Figure 4(a)). ${ }^{26}$

Spectra have been recorded in Figure 4(a) and (b) under different experimental settings. Thus, because the process reported in Figure $4(\mathrm{~b})$ occurs over a much shorter time range than that reported in Figure 4(a), in the first case it was not possible to accumulate the same number of spectra as for Figure 4(a), resulting in an apparent different noise degree.

Therefore, the difference observed for the time evolution of CD spectra can be explained by assuming that MMP-2 unwinds the triple helix from the beginning of the substrate degradation process. This variation should be associated with the preferential interaction of MMP-2 with collagen I through the fibronectin-like domain (corresponding to the CBD), absent in MMP-8 and in ectMMP-14
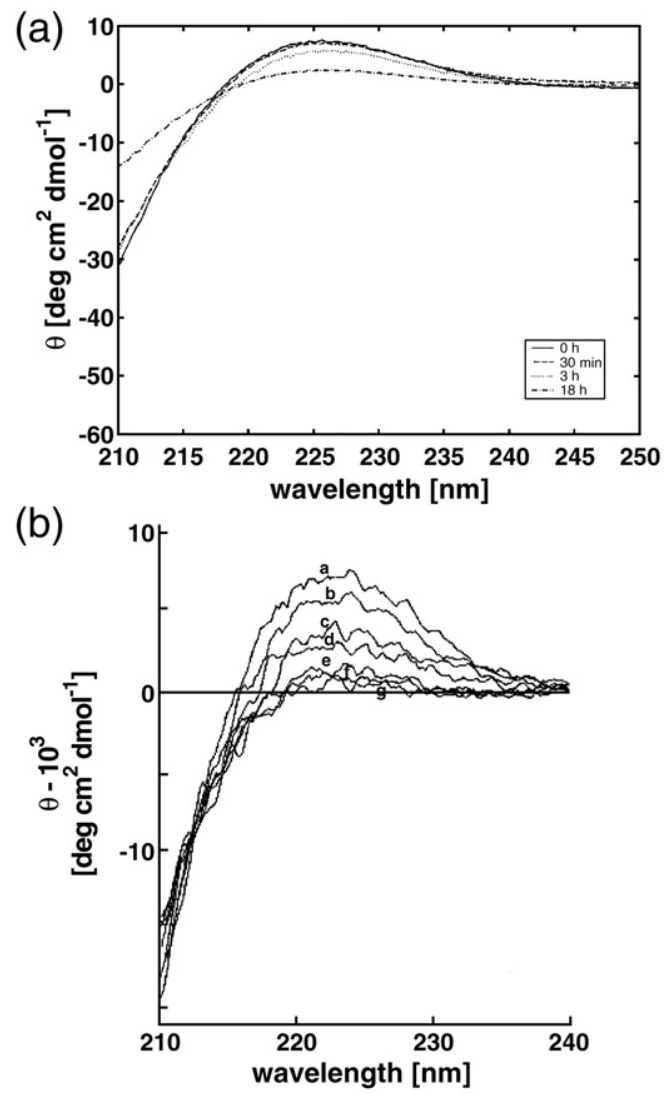

Figure 4. Circular dichroism spectra of agarose gelentrapped collagen I at different time intervals after exposure to $10 \mathrm{pM}$ ectMMP-14 (a) and $10 \mathrm{pM} \mathrm{MMP-2} \mathrm{(b)}$ at $37^{\circ} \mathrm{C}, \mathrm{pH}$ 7.3. In (a) time intervals are indicated, while in (b) time intervals are as follows: (a) $0 \mathrm{~min}$, (b) $30 \mathrm{~min}$, (c) $60 \mathrm{~min}$, (d) $120 \mathrm{~min}$, (e) $240 \mathrm{~min}$, (f) $360 \mathrm{~min}$, (g) $480 \mathrm{~min}$. It must be remarked that spectra reported in (b) are the averages of only two spectra because of the kinetics. For further details, see the text. 
(which instead should preferentially interact with collagen I through the hemopexin-like domain) and that only the interaction with the fibronectin-like domain induces a gross alteration of the triple helical structural arrangement, which is then followed by substrate cleavage and degradation (Figure 4(b)).

\section{Is cleavage needed to induce the triple helix unwinding of collagen I by MMP-2?}

To understand the MMP-2 collagen interaction itself as well as the subsequent effect on triple helix unwinding, we performed CD experiments employing fully inhibited MMP-2.

Batimastat was employed as inhibitor and the full inhibition of MMP-2 was validated with a synthetic substrate, but it was further reinforced by the observation (reported in Figure 1, lane 4) that after $3 \mathrm{~h}$ of incubation at $37^{\circ} \mathrm{C}$ with inhibited $2 \mu \mathrm{M}$ MMP2 no degradation of collagen I was detected. Over the same period at the same temperature, $10 \mathrm{pM}$ active MMP-2 brings about an extensive degradation of collagen I (see Figure 1, lane 6).

As shown in Figure 5, a variation of the CD spectra of gel-entrapped collagen I is detected even in the presence of fully inhibited MMP-2, indicating that the triple helix unwinding activity is present even in the absence of proteolytic cleavage. We carried out the experiment both in the presence of a substoichiometric amount of inhibited MMP-2 (1:10 ratio between MMP-2 and collagen I) and in the presence of a 2-fold excess of inhibited MMP-2 over collagen. The extent of triple helix unwinding (as indicated by the disappearance of the ellipticity at $223 \mathrm{~nm}$, see Figure 5) indeed is dependent on MMP2 concentration, because in the presence of substoichiometric amounts of MMP-2 only a partial unwinding can be observed (see spectrum $b$ in Figure 5), while an excess of MMP-2, which guarantees binding saturation of the high-affinity site on collagen I (see $K_{\mathrm{m}}$ for the $\alpha-1$ chain in Table 1), ${ }^{41}$

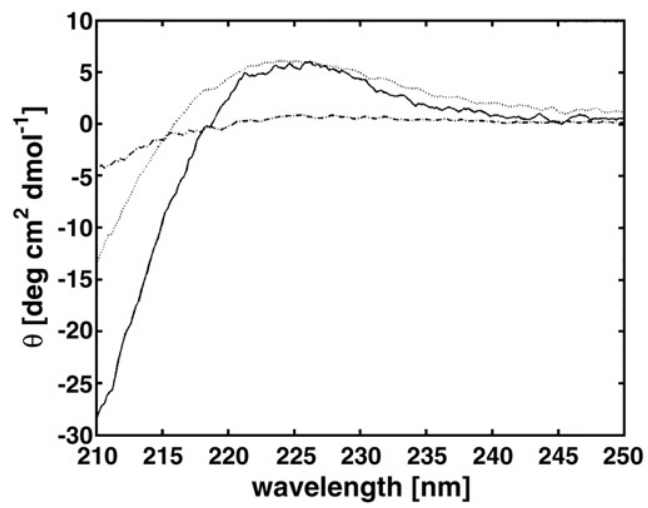

Figure 5. Circular dichroism spectra of agarose gelentrapped collagen I at a concentration of $1 \mu \mathrm{M}$ at $37^{\circ} \mathrm{C}$ (dotted spectrum), after the addition of $0.2 \mu \mathrm{M}$ MMP-2 fully inhibited by Batimastat (solid spectrum), and after the addition of $2 \mu \mathrm{M}$ MMP-2 fully inhibited by Batimastat (dashed spectrum). For further details, see the text. brings about an almost complete unwinding of the triple helix (see spectrum $c$ in Figure 5). These ellipticity changes occur immediately after the addition of inhibited MMP-2 and no further spectral change is observed even after $2 \mathrm{~h}$ from the addition, ensuring that (i) binding equilibrium is attained and (ii) no proteolysis of collagen is taking place. The observation that fully inhibited MMP-2 induces a dramatic decrease of ellipticity immediately after addition, whereas $10 \mathrm{pM}$ active $\mathrm{MMP}-2$ requires several hours for an analogous effect, indicates that: (i) the effect reported in Figure 5 (where the enzyme/substrate ratio is 1:10 for spectrum $b$ and $2: 1$ for spectrum $c$ ) is due to the binding (without substrate cleavage) of one molecule of MMP-2 for each molecule of triple helical collagen I; and (ii) the phenomenon observed in Figure 4(b) (where the enzyme/substrate ratio is $1: 10^{5}$ ) is due to the progressive binding and cleavage of triple helical collagen I by a minute amount of enzyme molecules, which must undertake the turnover process several times to bring about an observable unwinding. As a whole, this set of observations represents clear evidence that binding of collagen I by MMP-2 is enough to induce the unwinding of the triple helical assembly, with a behavior completely different from that observed in the case of MMP- $8^{26}$ and ectMMP14 (data not shown). The unwinding of the collagen I triple helix observed in Figure 5 is also confirmed by the fact that collagen I, which is still intact after $3 \mathrm{~h}$ of incubation at $37^{\circ} \mathrm{C}$ with $2 \mu \mathrm{M}$ fully inhibited MMP-2 (see Figure 1, lane 4), is instead more sensitive to trypsin degradation (see Figure 1, lane 5).

\section{Ex vivo collagenolytic activity of MMP-2}

To validate the powerful collagenolytic activity of MMP-2 we carried out a cleavage test model against Achilles mouse tendon, which is predominantly made of collagen I. ${ }^{42}$ Notably, it turned out that MMP-2 (10 pM active enzyme) retains its collagenolytic activity even against intact fibers of collagen. The strong-banded fibrils and microfibrils displayed by the control samples show that the fibers examined possess a strong substructure (see Figure 6(a) and (b)). Figure 6(c) and (d) illustrates the micrographs referring to the fibers exposed overnight to MMP-2 activity. Experimental conditions of examined samples are comparable to those employed for the kinetic experiments, unequivocally demonstrating a relevant proteolytic activity of MMP-2 against intact fibers of collagen I.

\section{Discussion}

The mechanism of collagen I processing by different MMPs is crucial for understanding the remodeling of the extracellular matrix, because fibrillar collagen is a major component of the architecture $^{1}$ and its degradation is heavily involved in growth factor-induced angiogenesis. ${ }^{43}$ Interestingly, the melting temperature $\left(T_{\mathrm{m}}\right)$ of collagens is 

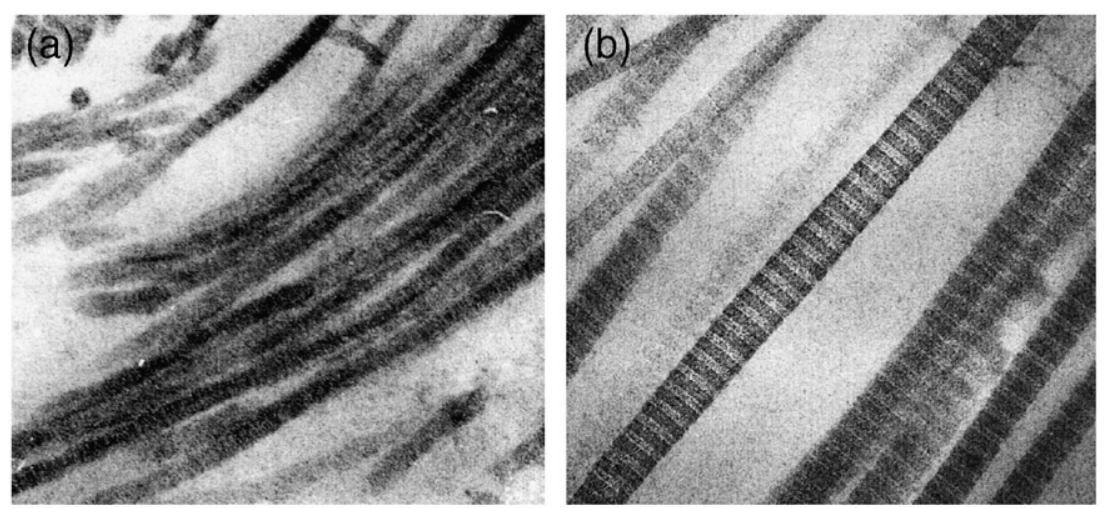

(c)
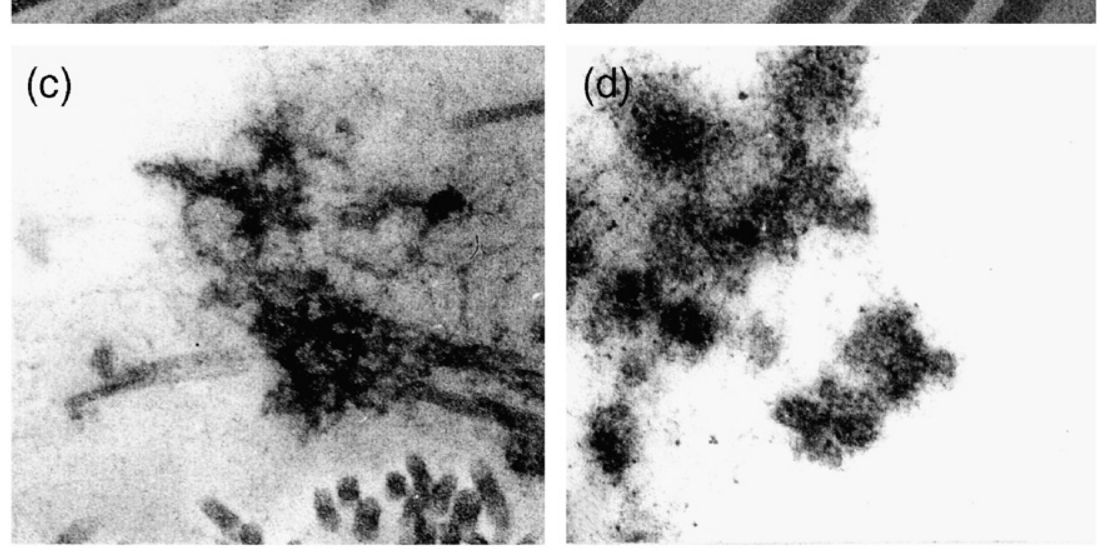

Figure 6. Transmission electron micrographs of the Achilles' tendons from 6-week-old mice. (a) and (b) Collagen fibers not exposed to the MMP-2 action; (c) and (d) collagen fibers treated with MMP2 . For (a) and (c) the optical magnification is $\times 28,500$; for (b) and (d) the optical magnification is $\times 52,000$. For further details, see the text.

related to the content of amino acids (i.e., proline and hydroxyproline). Thus, differences in the amino acidic content of two types of $\alpha$ chains (i.e., $\alpha-1$ and $\alpha-2$ chains $)^{44}$ should be reflected in different conformational stabilities within the triple helix, casting some light on the different mechanisms for the various MMPs.

Soluble fibrillar collagen I has been shown first to be enzymatically processed by collagenases (i.e., MMP-1, MMP-8, and MMP-13), ${ }^{25,45}$ but also by gelatinase A MMP-2, ${ }^{46}$ gelatinase B MMP-9, 47 and by MMP-14. ${ }^{48}$ In particular, because MMP-14 is a membrane-bound MMP, its ectodomain (ectMMP14) is also active on collagen I. ${ }^{14}$ In any event, it is very important to outline that the present paper clearly shows that cleavage of native fibrillar collagen I by gelatinase A actually takes place in fibers from mouse tendon, as observed by electron microscopy (see Figure 6), reinforcing the idea that it may occur as well under in vivo conditions.

However, since the size of the triple helical collagen I is exceedingly large to be accommodated in the active site of either of these enzymes, ${ }^{3,28-30}$ interaction and cleavage must occur through a series of steps, which allow the cleavable peptide bond to come into close contact with the active site. In this respect, it has been proposed that collagenases, such as MMP-1 and MMP-8, might interact with one chain at a time, ${ }^{21,25}$ locally unwinding the triple helix, probably through the hemopexin-like domain, because its absence brings about a drastic change in the way collagen I is processed. ${ }^{27}$ MMP-14 as well appears to interact with collagen I preferentially through the linker-hemopexin-like domain, which also seems crucial for the cleavage event, $23,49,50$ even though the mechanism has been proposed to be somewhat different from that of collagenases. ${ }^{23,51}$ On the other hand, even though a MMP-2 mutant (missing the fibronectin-like domain) retains the capability of cleaving collagen, ${ }^{17}$ MMP-2 does not seem to interact with fibrillar collagen I through its hemopexin-like domain. ${ }^{52}$ In fact, the fibronectinlike domain appears to be the preferential interaction site, probably inducing some conformational change, as from circular dichroism employing the CBD alone ${ }^{23}$ and fully inhibited MMP-2 (see Figure $5)$. The driving force exerted by the CBD of MMP$2,23,51$ which is not present in other MMPs, raises the question of whether the mechanism of triple helicase activity of MMP-2 must be fundamentally different from that of collagenases.

The answer to this question comes from circular dichroism observations, where a drastic difference can be observed for the interaction of MMP-8 and ectMMP-14 with triple helical collagen I compared with MMP-2. Thus, no meaningful alteration of the triple helical assembly of collagen I is observed upon binding only with MMP- ${ }^{26}$ as well as with ectMMP-14 (see above), whereas a disruption of the triple helix assembly is observed upon the addition of MMP-2 fully inhibited by Batimastat (in an enzyme concentration-dependent fashion for comparable concentrations of substrate and enzyme; see Figure 5). These results clearly indicate that while binding of the hemopexin-like domain of MMP-8 or of ectMMP-14 does not bring about any substantial alteration of the triple helix (if no subsequent cleavage occurs), the interaction of MMP-2 alone through its fibronectin-like domain is sufficient to induce an extended and widespread unwinding of 
fibrillar collagen I, even in the absence of any cleavage of the substrate. Furthermore, on the basis of the concentration of inhibited MMP-2 (i.e., $2 \mu \mathrm{M}$, which is enough only for binding to the $\alpha-1$ chain; see $K_{\mathrm{m}}$ in Table 1 ), we can extend our interpretation, claiming that interaction of the fibronectin-like domain of MMP-2 with the $\alpha-1$ chain of fibrillar collagen I is sufficient to induce an overall unwinding of a large portion of the triple helix. On the basis of such considerations and observations, the present data of MMP-2 suggest a preferential interaction of the fibronectin-like domain, which is not present in ectMMP-14 and MMP-8, with the $\alpha-1$ chain of fibrillar collagen, a conclusion that is also in line with previous observations on the interaction of the whole fibronectin molecule with fibrillar collagen I. ${ }^{53}$

This difference among the three MMPs investigated for the collagen I triple helix binding mechanism is also reflected in the catalytic parameters (see Table 1), because MMP-2 interacts preferentially with the $\alpha-1$ chain (as shown from the very low value for $K_{\mathrm{m}}$; see Table 1$)$, while it cleaves preferentially the $\alpha-2$ chain (as shown from the much higher value of $k_{\text {cat; }}$ see Table 1 ). Conversely, MMP-8 and ectMMP-14 appear instead to process the two types of chains of fibrillar collagen I in a similar fashion, without showing any preference for either chain (as shown from the values of $K_{\mathrm{m}}$ and $k_{\text {cat }}$ for $\alpha-1$ and $\alpha-2$ chains; see Table 1). Therefore, these data seem to suggest that as long as the triple helix is maintained, no preferential enzymatic action is exerted on the two types of chains. On the other hand, whenever unwinding occurs, as with binding by MMP-2, the two types of chains display a drastically different propensity for proteolytic attack. The peculiar behavior of MMP-2 in processing the two types of chains of collagen is not surprising, because binding of the CBD (and thus likely also of the fibronectinlike domain) has been proposed (and is substantiated by the present data) to involve simultaneously two collagen chains, ${ }^{54}$ exerting triple helicase activity. ${ }^{15}$ The distinctive features of MMP-2 action on collagen I, which requires a preliminary unwinding of the triple helix, also account for the dramatic temperature dependence of MMP-2 cleavage activity on collagen I (which is almost vanishingly small at $\left.25^{\circ} \mathrm{C}\right) .{ }^{45}$ Thus, unlike collagenases and ectMMP14 , the activation free energy for the enzymatic processing of the triple helical collagen I by MMP-2 includes (beside the intrinsic temperature dependence for the proteolytic activity) $)^{37}$ a contribution from the unwinding of the triple helix of collagen I. As a matter of fact, the energy needed for the unwinding by MMP-2 is much higher at $25^{\circ} \mathrm{C}$ (thus slowing down the process) than at $37{ }^{\circ} \mathrm{C}$, where the triple helical arrangement is much looser. This observation is in line also with recent data on the enzymatic activity of several MMPs toward synthetic triple helical substrates, ${ }^{24}$ where MMP-2 turned out to be less active than MMP-8 and MMP-14 toward tighter and more thermostable substrates.
As a whole, like at $25^{\circ} \mathrm{C},{ }^{20-24}$ at $37^{\circ} \mathrm{C}$ a dramatic difference can be outlined between the proteolytic mechanism of MMP-8 (as a possible prototype of the collagenases action) and ectMMP-14 on one side and MMP-2 (as a possible prototype of gelatinases) on the other side, and this appears mostly related to the interaction mode with collagen I. Thus, MMP-8 and ectMMP-14 interact through the hemopexin-like domain, without significantly perturbing the triple helical assembly of fibrillar collagen, likely processing one chain at a time in a similar way (as shown by the closely similar catalytic parameter for the two chains; see Table 1). On the other hand, MMP-2 actually induces a denaturation of fibrillar collagen (likely binding the $\alpha-1$ chains; see Table 1) and then it enzymatically processes fibrillar collagen, cleaving preferentially the $\alpha-2$ chain (see Table 1) and suggesting that the unwinding induced by the binding of MMP-2 is possibly the rate-limiting step for the proteolytic attack of collagen I by MMP-2 (Figure 7).

Structural interpretation of the preferential enzymatic action on the $\alpha-2$ chain by MMP-2 (see Table 1 ) is not straightforward, because we do not know the detailed structural characteristics of the two different chains. However, even though both amino acid sequences of type I collagen are homologous, the $\alpha-2$ chain is more hydrophobic and has a lower amino acid content than the $\alpha-1$ chain $^{1}$ and it has been shown that the higher amino acid content of $\alpha-1$ chain gives a larger contribution to the conformational stability of the collagen triple helical structure. 4,55 Therefore, a purely speculative consideration might try to correlate the higher hydrophobicity and the lower amino acid content of $\alpha-2$ chains to a higher value of $K_{\mathrm{m}}$ and a faster $k_{\text {cat }}$ (see Table 1).

As a concluding remark, binding through the hemopexin-like domain (as for MMP-8 and ectMMP-14) brings about a topologically specific interaction, accompanied by a very limited and mild alteration of the triple helix assembly, and it underlies similar processing for the two types of chains forming the collagen triple helix. On the other hand, binding through the fibronectin-like domain (as for MMP-2) grossly perturbs the triple helical structure, leading to more extended and chainspecific substrate cleavage. These functional differences appear to reflect and can accounted for the distinct role of these MMPs "in vivo," which is tissue modeling for collagenases (such as MMP-8 and in part ectMMP-14), while gelatinases (such as MMP-2) seem to be more involved in disruptive events related to pathological processes.

\section{Materials and Methods}

\section{Materials}

Purity of MMP-2, MMP-8, and ectMMP-14 proenzymes, (R\&D Systems, London, UK) wasbeen measured by SDSPAGE described by Laemmli. ${ }^{56}$ After the gels were run, 
MMP2

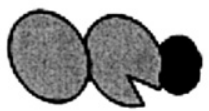

$\$ 00000$

$\downarrow$
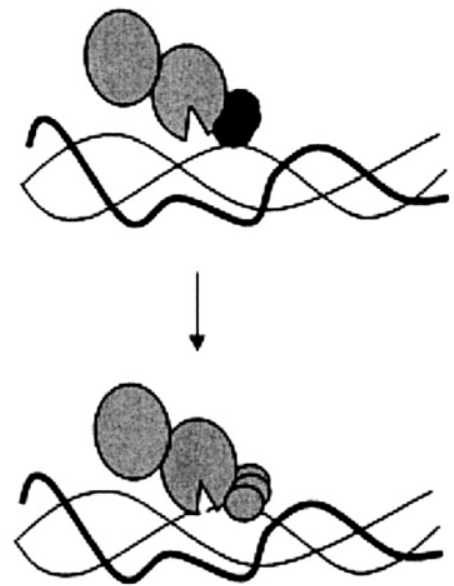

MMP8 or ect-MMP14

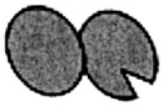

$\$ 00000$

$\downarrow$

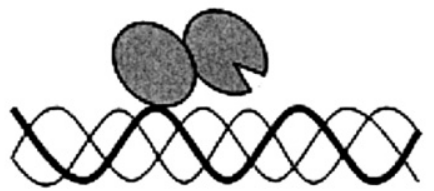

$\downarrow$

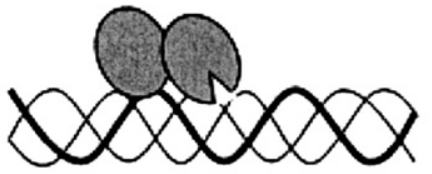

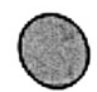

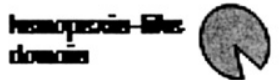

contutic

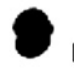

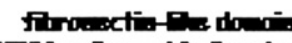

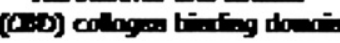

Figure 7. (Left) A molecular sketch of the possible steps for the cleavage of triple helical fibrillar collagen I by MMP-2, showing the interaction through the fibronectinlike domain and the unwinding of the triple helix before cleavage. (Right) A molecular sketch of the possible steps for the cleavage of triple helical fibrillar collagen I by MMP-8 and/or ectMMP-14, showing the interaction through the hemopexin-like domain, which leaves intact the triple helix before the cleavage. Thin lines refer to $\alpha-1$ chains of the triple-helical collagen I and thick lines refer to the $\alpha-2$ chain. For further details, see the text. they were stained using a silver staining kit (Bio-Rad, Hercules, CA, USA). The broad spectrum protein markers (Bio-Rad) were used as molecular mass standards.

The stability of each MMP was checked by zymography (employing as substrates either gelatin and collagen), as follows: $2 \mu \mathrm{l}$ of MMP in collagen solution was mixed with a 5 -fold excess of sample buffer $(0.25 \mathrm{M}$ Tris- $\mathrm{HCl}, 0,8 \%$ SDS, $10 \%$ glycerol, and $0.05 \%$ bromophenol blue) and run on $12 \%$ SDS-PAGE containing either $1 \mathrm{mg} / \mathrm{ml}$ gelatin or bovine type I collagen as described previously. ${ }^{56}$

Trypsintpch and soybean trypsin inhibitor were purchased from Sigma (St. Louis, MO, USA). The quenched fluorogenic substrates employed for activity assays were (7-methoxycoumarin-4-yl)acetyl-Pro-cyclohexylalanineGly norvaline-His-Ala-N-3-(2,4-dinitrophenyl)-L-2,3-diaminopropionyl- $\mathrm{NH}_{2}$ and (7-methoxycoumarin-4-yl) acetyl-Pro-Leu-Gly Leu-N-3-(2,4-dinitrophenyl)-L-2,3diaminopropionyl-Ala-Arg- $\mathrm{NH}_{2}$, purchased from Calbiochem (La Jolla, CA, USA). Batimastat (BB-94), a peptidomimetic inhibitor, which stoichiometrically inhibits MMPs, was kindly provided by British Biotech Pharmaceutical (Cowley, Oxford, UK)

Acid-soluble calf collagen I, purchased from Sigma, was dissolved overnight at a concentration of $1 \mathrm{mg} / \mathrm{ml}$ in $0.1 \mathrm{M}$ acetic acid solution at room temperature. Afterward, the suspension was centrifuged at 10,000 $\mathrm{g}$ for $1 \mathrm{~h}$ and the supernatant, containing the dissolved collagen, was used. We also used rat-tail tendon type I collagen, isolated by standard ammonium sulfate precipitation procedures from the tail tendons of 12-week-old Wistar rats, as previously described by others in detail. ${ }^{52,57}$
The amount of collagen has been quantified as described, ${ }^{58}$ using soybean trypsin inhibitor as a reference. The triple helical nature of collagen has been checked by circular dichroism and confirmed by the absence of collagen degradation in trypsin control assays at an enzyme/ substrate ratio of $1: 10$ over $3 \mathrm{~h}$ at $28{ }^{\circ} \mathrm{C}$, as described previously. ${ }^{23}$ In order to validate that collagen retains its properties, even after having been exposed to $37^{\circ} \mathrm{C}$ for $24 \mathrm{~h}$, we brought the sample back to $28^{\circ} \mathrm{C}$ and repeated trypsin control assays: no degradation was observed under these conditions (see Figure 1, lane 2). Lyophilized collagen was stored at $-80{ }^{\circ} \mathrm{C}$, and stock solutions were prepared as needed. No significant difference in the triple helix content and stability was detected between the two preparations of collagen after solubilization.

Achilles' tendons, surgically extracted from young mice (manipulated as described by the ethical guidelines), were used for the electron microscopy experiments.

\section{Methods}

\section{Enzymatic assays}

Recombinant human MMP-2 and MMP-8 proenzymes were activated by incubating $0.1 \mathrm{mg} / \mathrm{ml}$ progelatinase solution with $0.25 \mathrm{mM}$ of aminophenyl mercuric acid (Sigma) at $37^{\circ} \mathrm{C}$ for $30 \mathrm{~min}$. The activation of pro-ectMMP14 was performed using $5 \mu \mathrm{g} / \mathrm{ml}$ trypsintpch at $25{ }^{\circ} \mathrm{C}$. Trypsin activity was quenched using $50 \mu \mathrm{g} / \mathrm{ml}$ soybean trypsin inhibitor.

The concentration of activated MMP was determined by classic fluorimetric assay, as described, ${ }^{59}$ following the 
progressive decrease of hydrolysis (upon the addition of Batimastat (BB-94)) of the quenched fluorogenic substrate (7-methoxycoumarin-4-yl)acetyl-Pro-cyclohexylalanineGly norvaline-His-Ala- $N-3-(2,4-d i n i t r o p h e n y l)-L-2,3-d i a-$ minopropionyl- $\mathrm{NH}_{2} \quad\left(\lambda_{\text {exc }}=325 \mathrm{~nm}, \lambda_{\mathrm{em}}=398 \mathrm{~nm}\right)$ for MMP-8 and (7-methoxycoumarin-4-yl)acetyl-Pro-LeuGly Leu-N-3-(2,4-dinitrophenyl)-L-2,3-diaminopropionyl-Ala-Arg- $\mathrm{NH}_{2}\left(\lambda_{\mathrm{exc}}=325 \mathrm{~nm}, \lambda_{\mathrm{em}}=393 \mathrm{~nm}\right)$, which is resistant to trypsin action, for MMP-2 and ectMMP-14. All measurements were performed at $37^{\circ} \mathrm{C}$ using a solution of $50 \mathrm{mM}$ Tris- $\mathrm{HCl}, 0.1 \mathrm{M} \mathrm{NaCl}, 10 \mathrm{mM} \mathrm{CaCl}_{2}$ plus $0.05 \%$ Brij 35 (polyoxyethylenelaurylether) buffered at pH 7.3.

\section{Determination of kinetic and thermodynamic parameters}

Kinetic analyses of $\alpha$-chain degradation by MMP-2, MMP-8, and ectMMP-14 were performed in $50 \mathrm{mM}$ Tris$\mathrm{HCl}, 0.1 \mathrm{M} \mathrm{NaCl}, 10 \mathrm{mM} \mathrm{CaCl}{ }_{2}$, at $\mathrm{pH}$ 7.3, employing different concentrations of bovine collagen I (spanning between 0.3 and $3.2 \mu \mathrm{M}$ ). Activated MMP was then added to the solutions (10 pM final concentration). It is worth stressing that MMPs alone are not stable after prolonged incubation at $37^{\circ} \mathrm{C}$; however, their stability is significantly increased in the presence of the substrate, as also reported by others. ${ }^{19,60}$

For each collagen concentration, mixtures were kept at $37^{\circ} \mathrm{C}$ and small aliquots were harvested at different time intervals. Reactions were stopped by the addition of SDSPAGE loading buffer containing $20 \mathrm{mM}$ EDTA and frozen to $-80{ }^{\circ} \mathrm{C}$ until used. Aliquots in the reducing sample buffer were separated on 7.5 or SDS-(10\%) PAGE gels and stained using either (i) the silver staining method or (ii) $0.5 \%$ Coommassie blue and then destained until substrate bands were clearly visible. The Coommassie blue electrophoretic spots, corresponding to different aliquots at different time intervals, were analyzed by a laser densitometer (LKB 2202 UltraScan) and their intensity was calibrated (to obtain concentration values) using standard substrate solutions. For each $\alpha$-chain (i.e., $\alpha-1$ and $\alpha-2$ chains) the substrate disappearance rates were derived at each collagen concentration employed.

The initial velocities were measured within $1 \mathrm{~h}$, that is the time period during which various MMPs were checked to be stable. Within this time interval, the degradation rate was constant and less than $10 \%$ of the substrate was degraded. This ensured a steady-state condition for the first cleavage step and was a prerequisite for the subsequent analysis step, which was carried out by simultaneous fitting of velocity experimental data described by both the Lineweaver-Burk equation (Equation 1(a)):

$$
\frac{E_{0}}{v}=\frac{K_{\mathrm{m}}}{k_{\mathrm{cat}}} \frac{1}{[S]}+\frac{1}{k_{\mathrm{cat}}}
$$

and the Eadie-Hofstee equation (Equation 1(b)):

$$
\frac{v}{E_{0}}=k_{\text {cat }}-\frac{v}{E_{0}[S]} K_{\mathrm{m}}
$$

where $E_{0}$ is the total enzyme concentration, $v$ is the actual rate (expressed as $\mathrm{mol} / \mathrm{s}), K_{\mathrm{m}}$ is the Michaelis-Menten equilibrium constant (expressed as mol), $k_{\text {cat }}$ is the ratelimiting step kinetic constant (expressed as $\mathrm{s}^{-1}$ ), and [S] is the substrate concentration. This procedure allowed more reliable values for the $k_{\mathrm{cat}}$ and $K_{\mathrm{m}}$ parameters to be obtained.

\section{Circular dichroism experiments}

CD spectra were recorded on a Jasco J-710 spectropolarimeter equipped with a thermostated cell holder and connected to a data station for signal averaging and processing. All spectra are averages of four scans (unless otherwise specified; see Figure 4(b)) and were recorded using quartz cells of 2-mm pathlength.

For the spectroscopic observations bovine collagen I was entrapped in agarose gel by mixing the solubilized protein to a $1 \%$ low-melting-point agarose solution at $\mathrm{pH}$ 3.0 and $37^{\circ} \mathrm{C}$. After being stirred rapidly the mixture was poured on a simple gel casting (Mini-protean II, Bio-Rad) and immediately cooled to obtain the gel. ${ }^{26}$ Final concentrations were $0.5 \%$ for agarose and $1 \mu \mathrm{M}$ for collagen. The thickness of the homogeneous gel was $1 \mathrm{~mm}$.

As a starting control, CD measurements were performed on samples of agarose gel soaked in sample buffer, which did not show any ellipticity, and data obtained were considered background. In addition, control CD measurements were also performed using gel-entrapped collagen at $\mathrm{pH}$ 3.0, as well as solubilized collagen I at the same $\mathrm{pH}$ and concentration conditions: no spectral difference between them was detected over the 200 to 250-nm spectroscopic range (a spectral region representative of the triple helical arrangement of collagen I) ${ }^{61}$ (data not shown), confirming that the collagen conformation was not altered by the gel entrapment procedure. This was also tested by incubating the agarose gel-entrapped collagen with $10 \mathrm{nM}$ trypsin for $3 \mathrm{~h}$ at $37{ }^{\circ} \mathrm{C}$ : no evidence of degradation was detected, as indicated by the lack of ellipticity changes at $223 \mathrm{~nm}$ over the incubation period (data not shown).

For kinetic experiments, slices of suitable size were cut from the homogeneous gel and kept overnight in buffer solution to reach the desired $\mathrm{pH}$ without the formation of aggregates and then used for CD measurements. ${ }^{26}$ Because collagen I has been reported to be somewhat thermally unstable in solution, ${ }^{34}$ gel-entrapped collagen samples of each proteolytic assay were monitored for as long as 3 days at $37^{\circ} \mathrm{C}$ and no significant change in CD spectra was detected in the absence of the enzyme (data not shown). The CD spectra so obtained are in agreement with those obtained for collagen-like peptides showing the characteristic triple helix maximum in the 223 to $225-\mathrm{nm}$ range. ${ }^{20}$ Therefore, we achieve a "quasi-native" model for evaluating structural arrangements during proteolytic processes on type I collagen. ${ }^{26}$ The concentrations of the agarose gel-entrapped collagen I were chosen so that the recorded CD spectra were obtained under conditions comparable to those employed for the fragmentations of soluble collagen I.

The kinetics of the unwinding of the triple helix was obtained in $0.2 \mathrm{M}$ Tris- $\mathrm{HCl}, 1 \mathrm{M} \mathrm{NaCl}, 10 \mathrm{mM} \mathrm{CaCl}_{2}$ solution at $37^{\circ} \mathrm{C}, \mathrm{pH} 7.3$, by recording the spectroscopic range (200-250 nm) as a function of time of incubation with the enzyme. Therefore, we derived the time evolution of the unwinding process reporting the molar ellipticity at 223-nm values of CD spectra recorded in the presence of different concentrations of each MMP species (spanning from $2 \mathrm{nM}$ to $2 \mu \mathrm{M}$ ).

As a control experiment, spectra of MMP (at the same concentration as that employed in the experiment) were recorded before every experiment in the same buffer solution. For all cases MMP ellipticity was vanishingly small (data not shown), ruling out the possibility of a mutual spectral distortion.

\section{Electron microscopy}

Achilles' tendons surgically extracted from 6-week-old mice were rinsed in phosphate-buffered saline and incubated overnight with active MMP-2 at $37^{\circ} \mathrm{C}$. Light 
microscopy and ultrastructural analysis were performed on samples fixed in $25 \%$ glutarhaldehyde in phosphatebuffered saline at $\mathrm{pH} 7.4$ and processed for transmission electron microscopy, as previously described. ${ }^{62}$ Two independent observers evaluated three different experiments.

\section{Acknowledgements}

Financial support from the Italian Ministery of University and Research (MIUR COFIN 2003058409) is gratefully acknowledged. We are also indebted to Dr C. M. Overall and Dr H. Nagase for several fruitful discussions.

\section{References}

1. Prockop, D. J. \& Kivirikko, K. I. (1995). Collagens: molecular biology, diseases, and potentials for therapy. Annu. Rev. Biochem. 64, 403-434.

2. Ottani, V., Marini, D., Franchi, M., Ruggeri, A. \& Raspanti, M. (2002). Hierarchical structures in fibrillar collagens. Micron, 33, 587-596.

3. Orgel, J. P. R. O., Irving, T. C., Miller, A. \& Wess, T. J. (2006). Microfibrillar structure of type I collagen in situ. Proc. Natl Acad. Sci. USA, 103, 9001-9005.

4. Jenkins, C. L., Bretscher, L. E., Guzei, I. A. \& Raines, R. T. (2003). Effect of 3-hydroxyproline residues on collagen stability. J. Am. Chem. Soc. 125, 6422-6427.

5. Shingleton, W. D., Hodges, D. J., Brick, P. \& Cawston, T. E. (1996). Collagenase: a key enzyme in collagen turnover. Biochem. Cell Biol. 74, 759-775.

6. Woessner, J. F. (1999). Matrix metalloproteinase inhibition. From the Jurassic to the third millennium. Ann. N.Y. Acad. Sci. 878, 388-403.

7. Brinckmann, J., Nees, C. M., Gaber, Y., Sobhi, H. Notbohm, H. \& Hunzelmann, N. (2001). Different pattern of collagen cross-links in two sclerotic skin diseases: lipodermatosclerosis and circumscribed scleroderma. J. Invest. Dermatol. 117, 269-273.

8. Brinckerhoff, C. E. \& Matrisian, L. M. (2002). Matrix metalloproteinases: a tail of a frog that became a prince. Nature Rev. Mol. Cell Biol. 3, 207-214.

9. Pilcher, B. K., Dumin, J. A., Sudbeck, B. D., Krane, S. M., Welgus, H. G. \& Parks, W. C. (1997). The activity of collagenase- 1 is required for keratinocyte migration on a type I collagen matrix. J. Cell Biol. 137, 1445-1457.

10. Zaho, W. G., Byrne, M. H., Boyce, B. F. \& Krane, S. M (1999). Bone resorption induced by parathyroid hormone is strikingly diminished in collagenaseresistant mutant mice. J. Clin. Invest. 103, 517-524.

11. Di Lullo, G. A., Sweeney, S. M., Körkkö, J., Ala-Kokko, L. \& San Antonio, J. D. (2002). Mapping the ligandbinding sites and disease-associated mutations on the most abundant protein in the human, type I collagen. J. Biol. Chem. 277, 4223-4231.

12. Sternlicht, M. D. \& Werb, Z. (2001). How matrix metalloproteinases regulate cell behavior. Annu. Rev. Cell Dev. Biol. 17, 463-516.

13. Overall, C. M. \& Lopez-Otin, C. (2002). Strategies for MMP inhibition in cancer: innovations for the posttrial era. Nature Rev. Cancer, 2, 657-672.

14. Itoh, Y. \& Seiki, M. (2006). MT1-MMP: a potent modifier of pericellular microenvironment. J. Cell Physiol. 206, 1-8.
15. Overall, C. M. (2002). Molecular determinants of metalloproteinase substrate specificity: matrix metalloproteinase substrate binding domains, modules, and exosites. Mol. Biotechnol. 22, 51-86.

16. Hotary, K. B., Allen, E. D., Punturieri, A., Yana, I. \& Weiss, S. J. (2000). Regulation of cell invasion and morphogenesis in a three-dimensional type I collagen matrix by membrane-type matrix metalloproteinases 1, 2, and 3. J. Cell. Biol. 149, 1309-1323.

17. Patterson, M. L., Atkinson, S. J., Knäuper, V. \& Murphy, G. (2001). Specific collagenolysis by gelatinase A, MMP-2, is determined by the hemopexin domain and not the fibronectin-like domain. FEBS Letters, 503, 158-162.

18. Hotary, K. B., Allen, E. D., Brooks, P. C., Datta, N. S., Long, M. W. \& Weiss, S. J. (2003). Membrane type I matrix metalloproteinase usurps tumor growth control imposed by the three-dimensional extracellular matrix. Cell, 114, 33-45.

19. Hurst, D. R., Schwartz, M. A., Ghaffari, M. A., Jin, Y., Tschesche, H., Fields, G. B. \& Sang, Q.-X. A. (2004). Catalytic-andecto-domains ofmembranetype1-matrix metalloproteinase have similar inhibition profiles but distinct endopeptidase activities. Biochem. J. 377, 775-779.

20. Lauer-Fields, J. L., Tuzinski, K. A., Shimokawa, K., Nagase, H. \& Fields, G. B. (2000). Hydrolysis of triplehelical collagen peptide models by matrix metalloproteinases. J. Biol. Chem. 275, 13282-13290.

21. Ottl, J., Gabriel, D., Murphy, G., Knäuper, V., Tominaga, Y. \& Nagase, H. (2000). Recognition and catabolism of synthetic heterotrimeric collagen peptides by matrix metalloproteinases. Chem. Biol. 7, 119-132.

22. Minond, D., Lauer-Fields, J., Nagase, H. \& Fields, G. B. (2004). Matrix metalloproteinase triple-helical peptidase activities are differentially regulated by substrate stability. Biochemistry, 43, 11474-11481.

23. Tam, E. M., Moore, T. R., Butler, G. S. \& Overall, C. M. (2004). Characterization of the distinct collagen binding, helicase and cleavage mechanisms of matrix metalloproteinase 2 and 14 (gelatinase A and MT1MMP): the differential roles of the MMP hemopexin c domains and the MMP-2 fibronectin type II modules in collagen triple helicase activities. J. Biol. Chem. 279, 43336-43344.

24. Minond, D., Lauer-Fields, J. L., Cudic, M., Overall, C. M., Pei, D. \& Brew, K. (2006). The roles of substrate thermal stability and $\mathrm{P}_{2}$ and $\mathrm{P}_{1}^{\prime}$ subsite identity on matrix metalloproteinase triple-helical peptidase activity and collagen specificity. J. Biol. Chem. 281,38302-38313.

25. Chung, L., Dinakarpandian, D., Yoshida, N., LauerFields, J. L., Fields, G. B., Visse, R. \& Nagase, H. (2004). Collagenase unwinds triple-helical collagen prior to peptide bond hydrolysis. EMBO J. 23, 3020-3030.

26. Marini, S., Fasciglione, G. F., De Sanctis, G., D'Alessio, S., Politi, V. \& Coletta, M. (2000). Cleavage of bovine collagen I by neutrophil collagenase MMP-8. Effect of $\mathrm{pH}$ on the catalytic properties as compared to synthetic substrates. J. Biol. Chem. 275, 18657-18663.

27. Gioia, M., Fasciglione, G. F., Marini, S., D'Alessio, S., De Sanctis, G. \& Diekmann, O. (2002). Modulation of the catalytic activity of neutrophil collagenase MMP-8 on bovine collagen I. Role of the activation cleavage and of the hemopexin-like domain. J. Biol. Chem. 277, 23123-23130.

28. Li, J., Brick, P., O’Hare, M. C., Skarzynski, T., Lloyd, L. F., Curry, V. A. et al. (1995). Structure of full-length 
porcine synovial collagenase reveals a C-terminal domain containing a calcium-linked, four-bladed beta-propeller. Structure, 3, 541-549.

29. Gomis-Ruth, F. X., Gohlke, U., Betz, M., Knäuper, V., Murphy, G., Lopez-Otin, C. \& Bode, W. (1996). The helping hand of collagenase-3 (MMP-13): 2.7 A crystal structure of its C-terminal haemopexin-like domain. J. Mol. Biol. 264, 556-566.

30. Morgunova, E., Tuuttila, A., Bergmann, U., Isupov, M., Lindqvist, Y., Schneider, G. \& Tryggvason, K (1999). Structure of human pro-matrix metalloproteinase-2: activation mechanism revealed. Science, $\mathbf{2 8 4}$ 1667-1670.

31. Perona, J. J., Tsu, C. A., Craik, C. S. \& Fletterik, R. J. (1997). Crystal structure of an ecotin-collagenase complex suggests a model for recognition and cleavage of the collagen triple helix. Biochemistry, 36, 5381-5392.

32. Lauer-Fields, J. L., Juska, D. \& Fields, G. B. (2002). Matrix metalloproteinases and collagen catabolism. Biopolymers, 66, 19-32.

33. Steplewski, A., Majsterek, I., McAdams, E., Rucker, E., Brittingham, R. J. \& Ito, H. (2004). Thermostability gradient in the collagen triple helix reveals its multidomain structure. J. Mol. Biol. 338, 989-998.

34. Briknarova, K., Gehrmann, M., Banayai, L., Tordai, H., Patthy, L. \& Llinas, M. (2001). Gelatin-binding region of human matrix metalloproteinase-2: solution structure, dynamics, and function of the COL-23 twodomain construct. J. Biol. Chem. 276, 27613-27621.

35. Leikina, E., Mertts, M. V., Kuznetsova, N. \& Leikin, S. (2002). Type I collagen is thermally unstable at body temperature. Proc. Natl Acad. Sci. USA, 99, 1314-1318.

36. Mallya, S. K., Mookhtiar, K. A., Gao, Y., Brew, K., Dioszegi, M., Birkedal-Hansen, H. \& van Wart, H. E. (1990). Characterization of 58-kilodalton human neutrophil collagenase: comparison with human fibroblast collagenase. Biochemistry, 29, 10628-10634.

37. Fasciglione, G. F., Marini, S., D'Alessio, S., Politi, V. \& Coletta, M. (2000). pH- and temperature-dependence of functional modulation in metalloproteinases. A comparison between neutrophil collagenase and gelatinases A and B. Biophys. J. 79, 2138-2149.

38. Helary, C., Foucault Bertaud, A., Godeau, G., Coulomb, B. \& Guille, M. M. (2005). Fibroblast populated dense collagen matrices: cell migration, cell density and metalloproteinases expression. Biomaterials, $\mathbf{2 6}$ 1533-1543.

39. McAnulty, R. J. \& Laurent, G. J. (1990). In Methods in Cartilage Research (Maroudas, A. \& Kuettner, K., eds), pp. 140-142, Academic Press, San Diego, CA.

40. Kuznetsova, N. \& Leikin, S. (1999). Does the triple helical domain of type I collagen encode molecular recognition and fiber assembly while telopeptides serve as catalytic domains? Effect of proteolytic cleavage on fibrillogenesis and on collagen-collagen interaction in fibers. J. Biol. Chem 274, 36083-36088.

41. Xu, X., Chen, Z., Wang, Y., Yamada, Y. \& Steffensen, B. (2005). Functional basis for the overlap in ligand interactions and substrate specificities of matrix metalloproteinases-9 and -2. Biochem. J. 392, 127-134.

42. Boot-Handford, R. P. \& Tuckwell, D. S. (2003). Fibrillar collagen: the key to vertebrate evolution? A tale of molecular incest. BioEssays, 25, 142-151.

43. Seandel, M., Noack-Kunnmann, K., Zhu, D., Aimes, R. T. \& Quigley, J. P. (2001). Growth factor-induced angiogenesis in vivo requires specific cleavage of fibrillar type I collagen. Blood, 97, 2323-2332.

44. Berisio, R., Vitagliano, L., Mazzarella, L. \& Zagari,
A. (2002). Crystal structure of the collagen triple helix model [(Pro-Pro-Gly)(10)](3). Protein Sci. 11, 262-270.

45. Inada, M., Wang, Y., Byrne, M. H., Rahman, M. U., Miyaura, C., Lopez-Otin, C. \& Krane, S. M. (2004). Critical roles for collagenase-3 (MMP13) in development of growth plate cartilage and in endochondral ossification. Proc. Natl Acad. Sci. USA, 101, 17192-17197.

46. Aimes, R. T. \& Quigley, J. P. (1995). Matrix metalloproteinase-2 is an interstitial collagenase. Inhibitorfree enzyme catalyzes the cleavage of collagen fibrils and soluble native type I collagen generating the specific 3/4- and 1/4-length fragments. J. Biol. Chem. 270, 5872-5876.

47. Allan, J. A., Docherty, A. J., Barker, P. J., Huskisson, N. S., Reynolds, J. J. \& Murphy, G. (1995). Binding of gelatinases $\mathrm{A}$ and $\mathrm{B}$ to type-I collagen and other matrix components. Biochem. J. 309, 299-306.

48. Ohuchi, I., Imai, K., Fujii, Y., Sato, H., Seiki, M. \& Okada, Y. (1997). Membrane type 1 matrix metalloproteinase digests interstitial collagens and other extracellular matrix macromolecules. J. Biol. Chem. 272, 2446-2451.

49. Knäuper, V., Cowell, S., Smith, B., Lopez-Otin, C., O'Shea, M. \& Morris, H. (1997). The role of the C-terminal domain of human collagenase-3 (MMP-13) in the activation of procollagenase-3, substrate specificity, and tissue inhibitor of metalloproteinase interaction. J. Biol. Chem. 272, 7608-7616.

50. d'Ortho, M. P., Will, H., Atkinson, S., Butler, G. S., Messent, A. \& Gavrilovic, J. (1997). Membrane-type matrix metalloproteinases 1 and 2 exhibit broadspectrum proteolytic capacities comparable to many matrix metalloproteinases. Eur. J. Biochem. 250, 751-757.

51. Tam, E. M., Wu, Y. I., Butler, G. S., Stack, M. S. \& Overall, C. M. (2002). Collagen binding properties of the membrane type- 1 matrix metalloproteinase (MT1MMP) hemopexin $C$ domain. The ectodomain of the 44-kDa autocatalytic product of MT1-MMP inhibits cell invasion by disrupting native type I collagen cleavage. J. Biol. Chem. 277, 39005-39014.

52. Wallon, U. M. \& Overall, C. M. (1997). The hemopexin-like domain (C domain) of human gelatinase $\mathrm{A}$ (matrix metalloproteinase-2) requires $\mathrm{Ca} 2+$ for fibronectin and heparin binding. Binding properties of recombinant gelatinase $\mathrm{A} \mathrm{C}$ domain to extracellular matrix and basement membrane components. J. Biol. Chem. 272, 7473-7481.

53. Guidry, C., Miller, E. J. \& Hook, M. (1990). A second fibronectin-binding region is present in collagen alpha chains. J. Biol. Chem. 265, 19230-19236.

54. Collier, E. E., Krasnov, P. A., Strongin, A. Y., BirkedalHansen, H. \& Goldberg, G. I. (1992). Alanine scanning mutagenesis and functional analysis of the fibronectin-like collagen-binding domain from human $92-\mathrm{kDa}$ type IV collagenase. J. Biol. Chem. 267, 6776-6781.

55. Xu, Y., Hyde, T., Wang, X., Bhate, M., Brodsky, B. \& Baum, J. (2003). NMR and CD spectroscopy show that imino acid restriction of the unfolded state leads to efficient folding. Biochemistry, 42, 8696-8703.

56. Laemmli, U. K. (1970). Cleavage of structural proteins during the assembly of the head of bacteriophage T4. Nature, 227, 680-683.

57. Chandrakasan, G., Torchia, D. A. \& Piez, K. A. (1976). Preparation of intact monomeric collagen from rat tail tendon and skin and the structure of the nonhelical ends in solution. J. Biol. Chem. 251, 6062-6067. 
58. Bradford, M. (1976). A rapid and sensitive method for the quantitation of microgram quantities of protein utilizing the principle of protein-dye binding. Anal. Biochem. 72, 248-254.

59. Knight, C. G., Willenbrock, F. \& Murphy, G. (1992). A novel coumarin-labelled peptide for sensitive continuous assays of the matrix metalloproteinases. FEBS Letters, 296, 263-266.

60. Nagase, H., Suzuki, K., Morodomi, T., Enghild, J. J. \& Salvesen, G. (1992). Activation mechanisms of the precursors of matrix metalloproteinases 1, 2 and 3. Matrix Suppl. 1, 237-244.

61. McBride, D. J., Jr, Choe, V., Shapiro, J. R. \& Brodsky, B. (1997). Altered collagen structure in mouse tail tendon lacking the alpha 2(I) chain. J. Mol. Biol. 270, 275-284.

62. Masuelli, L., Bei, R., Sacchetti, P., Scappaticci, I., Francalanci, P. \& Albonici, L. (2003). Beta-catenin accumulates in intercalated disks of hypertrophic cardiomyopathic hearts. Cardiovasc. Res. 60, 376-387.

Edited by M. Moody

(Received 21 October 2006; received in revised form 19 February 2007; accepted 21 February 2007) Available online 2 March 2007 